\title{
COMPLICATIONS IN CHILDREN WITH PEG TUBES AND NURSING CARE
}

\author{
Hatice Pars, Hacettepe University, Faculty of Nursing, Turkey
}

\begin{abstract}
Sufficient energy and essential nutrients is vital for normal growth and development in childhood. Ideally, Tube feeding in pediatric patients is beneficial and has positive effects in controlling and managing diseases and providing appropriate nutrition in children. However, it is important to prevent potential complications in postoperative patients, which can be classified into 5 groups: mechanic, gastrointestinal, metabolic, infectious, and pulmonary complications.
\end{abstract}

Mechanical complications: Tube obstruction, leakage from the tube edges, hyper- granulation, and buried bumper syndrome (BBS) are the most common mechanical complications after PEG tube placement.

Slow infusion of products with high calories or high-fiber contents, and adminis- tration of blended foods via tubes, increases the risk of obstruction. Furthermore, the administration of drugs without grinding and dissolving them well significantly increases the incidence of tube obstruction (Avitsland et al. 2006; Bankhead et al., 2009; El-Matary, 2008; Goldberg et al., 2010; Socia \& Friedman, 2011).

Regular irrigation is recommended to prevent tube obstruction. The irrigation procedure must be applied once every 4-8 hours in situations of continuous infusion and before and after each feeding in bolus feedings. The tube must be irrigated prior to and following drug administration. In case of any obstruction, irriga- tion with lukewarm water and consecutive application of mild pressure and aspiration can be performed. The guiding wires, cytology brushes, and an endoscopic retrograde cholangiopancreatography (ERCP) tube can also be used to open the obstruction (Bankhead et al., 2009; Friedman et al., 2014; Goldberg et al., 2010; Socia \& Friedman, 2011).

\section{Gastrointestinal Complications}

Gastrointestinal complications are the second most common complications of using PEG tubes. The most common complications are nausea, vomiting, increased gastric residues, abdominal distention, and diarrhea.

To prevent these complications, it is essential to regulate the administration rate of the nutritional formula to reach the target dose in several days, apply continuous infusion, prevent contamination, and administer the product at $68^{\circ} \mathrm{F}-75^{\circ} \mathrm{F}\left(20^{\circ} \mathrm{C}-24^{\circ} \mathrm{C}\right)$ (El-Matary, 2008; Friedman et al., 2014; Mahan et al., 2011).

\section{Corresponding author: hatice.saglamhs@gmail.com}

Pars, H., Çavuşoğlu, H. (2017). A literature review of Percutaneous Endoscopic Gastrostomy. Gastroenterology nursing.

This could be a place for your sources.
Metabolic complications: It may exist in relation to insufficient nutrition (deficiency of energy and protein intake, deficiency of micro-food, hyponatremia, hypokalemia, hypophosphatemia, hypoglycemia) and excessive nutrition (refeeding syndrome). This complication can be prevented by reducing the administration rate and osmolarity of the enteral product and by providing feeding with continuous infusion (El-Matary, 2008; Fröhlich et al., 2009)

Pulmonery complications: While lying in the supine position, swallowing difficulties, delayed gastric emptying time, and reflux can also contribute to pulmonary complications. Elevating the head $30^{\circ}-45^{\circ}$ during feeding, using prokinetic drugs in patients with delayed gastric emptying time, feeding with continuous infusion, and postpyloric placement of the tube (which decreases the risk of aspiration but does not completely eliminate it) are required for the prevention of these complications. The administration of water after feeding (the pressure in the lower esophagus decreases by washing with $10 \mathrm{ml}$ of water within 15 minutes following feeding) is also essential (Crosby \& Duerksen, 2005; El-Matary, 2008; Frohlich et al., 2009; Mahan et al., 2011; Soscia \& Friedman, 2011; Width \& Reinhard, 2009).

\section{Infectious Complicaitons:}

To asses peristomal skin integrity, comprehensive evaluation of peristomal skin (color, moisture, odor, erythema, temperature increase, maceration, and lesion) and stoma care using the correct care product are necessary (Goldberg et al., 2010; Rahnemai-Azar et al., 2014; Zulkowski, Ayello, \& Stelton, 2014). According to the literature, it is advisable to change the sterile dressing daily until granulation of the stoma canal has taken place, provide local disinfection (usually Days 1-7), and clean the site with additive-free pH 5.5 soap (Braegger et al., 2010; Itkın et al., 2011; Löser et al., 2005; Pedrón-Giner et al., 2013; Socia \& Friedman, 2011).

Nurses play a key role in implementing the nutritional plan of care for patients, including advocating for early commencement of enteral nutrition, assessment of calorie requirements, and initiating, titrating, and administering the feed. To sustain this important treatment successfully, nurses should effectively manage the therapeutic processes and postoperative care, recognizing possible complications. 